\title{
Research on Hierarchical \& Classified Teaching Model of Public Computer Course in University
}

\author{
X.G. HUO \\ Department of Computer Science, China Women's University, Beijing, China
}

\begin{abstract}
Public computer course of colleges and Universities under the traditional teaching pattern has been difficult to adapt to the demand of new situation. Aiming at some problems existing in current computer public basic course, the paper analyzes the necessity of the implementation of classification of stratified teaching model in Colleges and universities public computer course teaching, expounds the implementation scheme of a hierarchical classification of practical teaching. Finally, the paper points out some problems needing attention in the teaching of colleges and universities public computer course in hierarchical classification.
\end{abstract}

KEYWORD: Hierarchical \& Classified Teaching; Teaching Reform; Computer Public Course

\section{INTRODUCTION}

It has become the basic requirement of talents in 21 Century to master the basic knowledge of computers, the basic application of computer proficiency. Public computer course in higher school is an indispensable part of university teaching, but also college students must master the practical, very strong application course. With the cross disciplinary and integration between, on students' computer application ability of different majors are also put forward higher request and more specific, computer level become the prominent symbol to measure service quality and comprehensive ability of College students. Hierarchical teaching of university computer foundation teaching refers to the new students scientifically divided into several groups with similar levels of their respective groups and different teaching on the basis of computer knowledge and ability existing students, these groups can get the best development and improvement in classification strategy appropriate teachers and the interaction. Classification teaching university computer foundation teaching according to students of different majors, different computer courses for the elective, enable students to try to get an education suitable to their own development needs. The main body and the key is "teaching". Teaching is to impart knowledge as the foundation, the teacher to the student in a planned, step-by-step guide to its development, obtained in the master basic cultural knowledge and skills at the same time in the aspects of morality, intelligence, body, beauty, etc.. The hierarchical classification, namely the teacher clearly in the students' intelligence factors and non intelligence factors, the basic knowledge of the difference of the premise, the development of different teaching goals, set different teaching links, use different teaching methods, different teaching organization. The effective implementation of the organizational form of teaching, make the teaching effect to achieve optimal, will eventually benefit students. Hierarchical classification teaching of college computer basis teaching is the teaching structure of a multi-level and multi type, it accords with the teaching rules and the principle of teaching students in accordance with their aptitude, also the foundation, and the interest of students, professional development and adapt. This paper describes the scheme will be computer based, application and improvement of the three levels of teaching according to different school curriculum plan arrangement gradually implemented in the right term. Classification teaching arrangement in application and improve the implementation of hierarchical teaching, its teaching content according to different students of different elective course of computer specialty. At the same time, teachers in teaching through appropriate practical examples of the corresponding subject interspersed inspire students, to enhance the learning initiative of students. 


\section{THE NECESSITY OF COMPUTER PUBLIC COURSE HIERARCHICAL \& CLASSIFIED TEACHING}

With the China university enrollment year after year, there are obvious differences in college admission level of computer, coupled with the regional difference, computer courses in high school stage of the popularization of differences and other factors, lead to differences in the ability of students enrollment larger. But the present teaching form mostly stay in large classes make it rigidly uniform like teaching mode, namely, unified management, unified teaching and unified assessment, with students' levels of diversification does not suit completely. Teaching reform must therefore be humanized caixing.

It is beneficial to stimulate students' learning enthusiasm. Education experts say: students to learn new knowledge difficulty if slightly higher than the student's present degree of knowledge, is to stimulate the students' learning enthusiasm. If difficulty is too high, the students' knowledge structure and they have been unable to match, the students will gradually lose confidence, anxiety, loss of initiative learning difficulty; if too low, the students feel that learning is not challenging, also can produce anxiety and boredom, learning without enthusiasm, also can not receive better learning effect. Methods stratified teaching through the entrance of layered evaluation way to put the new students a level of ability to distinguish, enables the computer to similar levels of students into a group to go to, it can enable students to give full play to the enthusiasm of learning, do teach students in accordance with their aptitude in certain extent. The hierarchical teaching mode can according to the actual students of computer theory and practice level classes, can make the foundation is quite classmates, is conducive to the basis of good students deep knowledge, but also conducive to the weaker students step by step to learn computer knowledge, and achieve the same teaching objectives in different teaching plan after learning. Hierarchical teaching according to the level of knowledge, the ability to accept students of different levels, targeted, individualized, and help the students of different levels to determine the appropriate learning objectives and difficulty, progress within my ability, and pick up order, gradually improve.

It is beneficial to different students exert their professional expertise. With the development of computer technology increasingly deep into the various fields of expertise, specialized courses in many professional teaching has a lot of related to the knowledge and skills of computer, some professional class has the computer as an essential tool. Some professional development has to fully using the computer as a professional tool stage. It can be said: the application level of computer determines the ability and level of the students to solve the problems in the professional in the field of. Classification combining teaching professional students, students focus on computer applications, if implemented well, has great significance to the development of students' professional skills play and future career.

\section{THE IMPLEMENTATION STRATEGY OF THE HIERARCHICAL \& CLASSIFIED TEACHING MODEL}

First of all, need to build a scientific online evaluation system. The examination system need to be equipped with multiple sets of exam questions, questions covering computer culture foundation, office automation software, network infrastructure, the use of graph and image processing software to use content, questions can cover the choice questions, fill in the blanks, judge a problem, operation test etc. questions, score interval and time interval before evaluation evaluation unified set of evaluation questions. The new report, the school requires a unified notice freshmen at the specified time interval (can be the first week) to the specified open room to participate in the evaluation, in order to prevent cheating, evaluation process extraction questions need to ensure that students with a selected typical and different topics are fundamentally different. Through the basic knowledge of computer and automatic diagnostic evaluation of basic skills, can quickly, accurately obtain the evaluation result, which can determine the levels of students in grade.

It is very important to students stratification. Various levels must be appropriate, can according to the total number of students take the exam school delimit a proportion, such as identifying on top of $80 \%$ students belong to have basic skills recognized, can enter to further increase the learning level, this part of the students here called class A, but not enough $20 \%$ students based on the bottom, the need to develop independent according to the situation of students learning program, need to supplement the knowledge and skills step by step, this part of the students here called the B class. According to the guidelines of basic education of Education Department of information technology course, and the requirements of the "University non computer professional computer basic teaching guide", the teaching of university computer foundation can be divided into two levels: basic level, improve the level of application. At the same time, respectively, to formulate the corresponding teaching plans and teaching task: basic level teaching task is the basic computer knowledge and skills leveled up the middle school stage, mainly computer entry knowledge and basic operation. The students mainly 
to students in remote areas and family conditions are not good students. Teaching task of enhance level is to enable students to master the basic computer knowledge and basic skills necessary for the information society, so that students in the base of skilled operation computer, training students' ability of using computer skillfully handling problems. Teaching content mainly includes the operating system, commonly used software, safety knowledge, network application. Should implement the scouring the teaching process, it not only attaches importance to the teaching of basic knowledge and basic skills, but also attach importance to the ability of solving problems independently improve. But also to foster the students' ability of using computer processing of the professional or related fields, that is to say, the classification teaching is implemented in the level of teaching. Teaching content according to different students of different elective course of computer specialty. The school according to the school discipline school division divides the application of basic courses of computer application for the four categories, respectively is the education, economics and management, social sciences and arts, including social sciences category mainly according to the teaching of professional courses students computational thinking, mind maps, control structure and software graphics function; education mainly based on professional courses students education technology, computer assisted teaching, multimedia material processing, courseware making and the use of Author ware content; gold tube mainly according to the professional courses students teaching basic knowledge of database and Access database operation; art mainly according to the use of professional courses students teach some software such as Photoshop. The two levels mentioned above, students can jump according to their own learning in the process of learning.

Assessment strategies for students, need to be combined with the characteristics of basic computer knowledge, good students usual study summary and evaluation of process and operation in practice. The assessment and evaluation process evaluation, the evaluation stage, the case of integrating evaluation to students, so that not only makes the students' daily learning activities can be incorporated into the effective supervision and management, was able to conduct an objective and fair evaluation of students learning to get the real student learning effect. Among them, the process of evaluation, is refers to the teacher in the teaching process of comprehensive student classroom performance, the usual afterschool performance, team performance, and the unit assessment in four aspects to reflect the students' learning effect. Stage evaluation, refers to the teacher according to the teaching content, the formulation of multiple stages of teaching plan, and examine the students in different stages. Evaluation and refers to the unit test, mid-term and final exam results as the basis, to investigate the students' learning stage effect, and further provides some reference for floating stratification. Case evaluation, is a teacher of students learning attitude, learning methods, learning environment, physical and mental health of its full and comprehensive investigation and evaluation into the semester in. In a word level evaluation should be based on the difference and timeliness, encouraging principle, students should be to summarize, feedback, correction and improve the function.

In addition, the selection strategy of teaching materials, teachers need according to different levels of students write notes. The role of the teacher should also change accordingly, the teacher as the leadership, the student is the subject, people-oriented teaching process, students as the main role, teachers act as auxiliary, the guiding role. Teachers should enrich their knowledge and experience, enhance the sense of responsibility.

\section{SOME PROBLEMS NEED TO BE PAID ATTENTION TO}

Hierarchical classification teaching is a teaching method, the goal is to develop educational programs to students' level of training qualified talents to achieve different goals. But all is not a panacea, after all, the source of each student's language, culture, education and so on their own situation, teachers should fully realize the difference of students in the classroom theoretical teaching and experimental teaching in the process of teaching students in accordance with their aptitude, remember the idea, not across the board like in exactly the same attitude and measures of education students, but to the extent possible, in-depth understanding of each student, establish the teaching methods and adapt to the unique personality.

Teachers and experimental teachers need realtime to guide students to correctly understand the hierarchical teaching. After stratification, divided into lower level students might have an inferiority complex, coupled with the classmate levels close to, the lack of a mutual incentive environment, may influence the teaching effect. Therefore, to strengthen the students before the class to the correct understanding of the hierarchical teaching, solve puzzles them is very necessary. Teachers should let students know, the level of division is not immutable and frozen, through the efforts, the low level students can advance into the high levels of learning, thus eliminating the feelings of inferiority, and enhance the enthusiasm of learning, in order to achieve the desired teaching effects. Method.

For different levels of students, performance evaluation can not based on levels and delineated the 
score standard, and according to the need of basic level and growth of students in the learning process of the peacetime operations assessment achievement of the corresponding binding.

\section{SUMMARY}

Hierarchical classification teaching of public basic teaching of colleges and universities computer to break the traditional teaching mode which make it rigidly uniform like, with the actual needs of students and social demand for talents are adapted to the. The reform scheme not only has the enthusiasm of learning to teach students in accordance with their aptitude, stimulate the students, but also play to students professional skills and enhance the ability of teachers. Program specific implementation process, the formulation of reasonable evaluation strategy and design reasonable classification instance is the key. In addition, we must pay attention to the students' mentality guidance, and are fully aware of the student individual difference, teaching mechanism to adapt to the establishment of unique properties to phase. This scheme is still in the exploration, need to practice in the future to sum up experiences, and gradually improve, in order to meet the demands of the new situation.

\section{REFERENCES}

[1] XiangyunZhang, JiajunWang, HongfengSong The ordinary university public mathematics course classification layered model optimization research, Big learn several learn 2011, 4(27):11-14.

[2] Jeanette M.Wing. Coputational Thinking. Communications of the ACM, 2006, 49(3):33-35.

[3] LianggenHu, Kinds of big recruit students under the background of mathematics teaching reform, which is based on the experience of the Ningbo university, ningbo university press (education science edition), 2009, 4: 116119.

[4] LipingLi, XiangyunShen. Theory in the teaching of computer basic learning day classification teaching, Higher education Windows, February 2009:195-196.

[5] GuoqiangWang, Engineering mathematics educational reform the retrospect and prospect. China university mathematics, 2001, 4:35-37. 